\title{
Ducted propagation of chorus waves: Cluster observations
}

\author{
K. H. Yearby ${ }^{1}$, M. A. Balikhin ${ }^{1}$, Yu. V. Khotyaintsev ${ }^{2}$, S. N. Walker ${ }^{1}$, V. V. Krasnoselskikh ${ }^{3}$, H. St. C. K. Alleyne ${ }^{1}$, and \\ O. Agapitov ${ }^{3}$ \\ ${ }^{1}$ ACSE, University of Sheffield, Sheffield, UK \\ ${ }^{2}$ Swedish Institute of Space Physics, Uppsala, Sweden \\ ${ }^{3}$ LPC2E, Orleans, France
}

Received: 24 August 2011 - Revised: 1 September 2011 - Accepted: 12 September 2011 - Published: 26 September 2011

\begin{abstract}
Ducted propagation of whistler waves in the terrestrial magnetosphere-ionosphere system was discussed and studied long before the first in-situ spacecraft measurements. While a number of implicit examples of the existence of ducted propagation have been found, direct observation of ducts has been hampered by the low sampling rates of measurements of the plasma density. The present paper is based on Cluster observations of chorus waves. The ability to use measurements of the spacecraft potential as a proxy for high time resolution electron density measurements is exploited to identify a number of cases when increased chorus wave power, observed within the radiation belts, is observed simultaneously with density enchantments. It is argued that the observation of ducted propagation of chorus implies modification of numerical models for plasma-wave interactions within the radiation belts.
\end{abstract}

Keywords. Electromagnetics (Guided waves; Wave propagation) - Magnetospheric physics (Plasma waves and instabilities)

\section{Introduction}

Electron acceleration in the radiation belt is one of the unsolved problems of magnetospheric physics that has important implications for the security of many modern technological systems that rely on spacecraft operating at geosynchronous orbit. The large number of high energy electrons in the radiation belts pose a significant threat to satellites and thus represent one of the most significant hazards to spacecraft within the local geospace environment. Since a comprehensive physical model to describe this acceleration process has not yet been developed, the problem is approached using

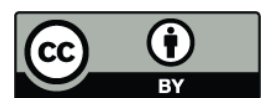

Correspondence to: M. A. Balikhin (m.balikhin@gmail.com) one of a number of numerical codes that include both the acceleration and precipitation of energetic electrons to model the evolution of the electron distribution. Many recent studies have focussed on local wave-particle interactions as the main cause of high fluxes of energetic electrons at geostationary orbit (e.g. Shprits et al., 2006; Shklyar and Kliem, 2006; Horne et al., 2007; Shklyar and Matsumoto, 2009; Shklyar, 2011a,b). One of the conclusions that follows from these studies is that chorus waves should play important role in the dynamics of high energy electrons. Chorus waves propagate in the whistler mode on the fast magnetosonic branch. They are observed in two frequency bands, a lower band at frequencies below half of the electron gyro-frequency and an upper band at frequencies above half of electron gyrofrequency. Recent observations by Cluster and THEMIS have been used to investigate the Poynting flux, distribution of wave vectors, and the scale of the source region (Santolik and Gurnett, 2003; Santolík et al., 2005; Agapitov et al., 2010). Many numerical models of the electron dynamics within the radiation belts are developed to investigate the interaction between chorus type waves and energetic electrons ( $\mathrm{Ni}$ et al., 2011). The basic algorithm exploited in this approach is as follows. A statistical analysis of plasma waves in the chorus frequency range is performed on the basis of in-situ measurements. Currently, the main source of this information comes from the CRRES satellite (Johnson and Kierein, 1992). A database of wave energy as a function of local time, L-shell, and $\mathrm{Kp}$ index was developed as the result of these statistical studies. The wave magnitudes are used to estimate the diffusion coefficients for quasilinear models for the energy exchange between waves and the plasma.

While the CRRES orbit was suitable for the experimental study of the plasma waves in the radiation belts, the interpretation of the limited number of parameters available in the CRRES wave data requires a number of assumptions about the properties of the observed waves. The most strict assumption is about the wave propagation direction. CRRES 
provides only electric field data to estimate the direction of the wave vectors. These directions are required both for the estimation of diffusion coefficients and to calculate the magnitudes of the magnetic component of the EM waves. Without experimental information about the wave propagation direction it is usually assumed that chorus waves propagate along the magnetic field. However, Cluster data have been used to show that the propagation of a significant number of chorus wave packets is actually oblique to the magnetic field (Santolík et al., 2009). This oblique propagation possess another problem for numerical models based on quasilinear diffusion. In the case of oblique propagation wave-particle interactions will involve not only waves propagating along a particular L-shell but also those waves that are propagating from neighbouring L-shells. Therefore the integration of the contribution to the diffusion coefficients from waves propagating from various L-shells should be calculated. The weighting of this integration must depend on the distribution of wave propagation directions. This information is not available from the CRRES data. Moreover, oblique propagation undermines the usual physical justification for the applicability of the quasilinear approximation itself. The quasilinear approach requires a random phase approximation. In classical quasilinear theory (Sagdeev and Galeev, 1969) a continuum of un-correlated plasma waves ensures that conditions for the random phase approximation are valid. The validity of this condition for discrete, highly structured chorus waves is not obvious. Usually the interaction between the chorus waves and the plasma has been justified on the basis of parallel propagation of the chorus waves. As the wave propagates along a magnetic field line it is subjected to multiple interactions between charged particles traveling between the two mirror points. Each interaction takes place at different spatial points providing the justification for random phase approximation. However, in case of oblique propagation the wave packet will leave a particular field line. This limits the number of times a wave packet can interact with particles on a particular field line and in the best case makes justification of the random phase approximation much more difficult.

However, all speculations mentioned above are only valid for non-ducted chorus. On the other hand, a chorus wave propagating in a duct formed along the magnetic field will not be able to travel far from the original field line and Lshell, even if the wave vector forms a large angle with the magnetic field. The idea of ducted whistlers was proposed as an interpretation of the very first observations of whistler waves. Storey (1953) interpreted occasional observations of long echo trains as indications of density inhomogeneities along the magnetic field lines that prevent the spreading of whistler energy. Helliwell et al. (1956) suggested that the discrete traces of whistler waves observed on spectrograms result from enhancements of the electron density aligned with the magnetic field. The theory of whistler trapping in field aligned density inhomogeneities was developed in the 1960s. Geometrical consideration of the refractive index sur- faces and ray tracing have been used to prove that whistler waves can be trapped in field-aligned columns of enhanced density providing that their frequency is below half the electron cyclotron frequency (e.g. Smith et al., 1960). In the case of field-aligned columns of lower density plasma whistler waves in the frequency ranges both below and above half cyclotron frequency can be trapped. The observation of ducts that are able to trap whistler waves have been hampered by the requirements of high resolution plasma data. However in a number of cases the study of the whistler wave propagation direction as a function of the location provided implicit indications that supported the existence of ducts (Burton and Holzer, 1974). In the present paper we exploit the use of the Cluster spacecraft potential as a high time resolution proxy measurement of the plasma density to provide information about changes in the plasma density that prove the existence of the ducted regime of propagation of chorus waves.

\section{Data and analysis}

The data used in this study were measured by the electric field instruments on board Cluster 4 on 23 August 2003 between 22:40 and 23:00 UT. During this period Cluster 4 was in the inner magnetosphere at a position (4.0, $1.7,-1.9) R_{\mathrm{E}}$ (L-shell $\sim 5$ ) and heading northwards and approaching perigee which occurred around midnight. The electric field sensors consist of four spherical probes, each attached to a $44 \mathrm{~m}$ wire boom. The output from these sensors, which cover the frequency range DC to $600 \mathrm{kHz}$, is processed by the Electric Field and Wave (EFW), Wideband Data (WBD), and WHISPER experiments which are controlled by the Digital Wave Processor (DWP) instrument (Woolliscroft et al., 1997). The EFW experiment (Gustafsson et al., 1997, 2001) samples the electric field at either $25 \mathrm{~Hz}(10 \mathrm{~Hz}$ filter) in normal science mode and $450 \mathrm{~Hz}$ $(180 \mathrm{~Hz}$ filter $)$ in burst science mode. In addition, EFW provides measurements of the probe to spacecraft potential sampled $5 \mathrm{~Hz}$.

The WBD experiment (Gurnett et al., 1997) directly records the high frequency waveform from a single pair of electric field probes (or the magnetic search coil if commanded). The instrument can operate in a number of different modes. The data presented in this paper was continuously recorded at a sampling rate of $27.4 \mathrm{kHz}$, with 8 bits resolution. In this particular operation mode, WBD cycles its sampling of both the electric field for a period of around $50 \mathrm{~s}$ followed by a short period of around $4 \mathrm{~s}$ when it samples the magnetic field from the search coil magnetometer. Automatic gain control allows a large dynamic range $(\sim 120 \mathrm{~dB})$ to be recorded however, it also implies that the general background level will be raised if large amplitude waves are detected.

WHISPER (Waves of High frequency and Sounder for Probing of Electron density) (Décréau et al., 1997) is a 
relaxation sounder and high frequency wave analyser covering the frequency range $4 \mathrm{kHz}$ to $80 \mathrm{kHz}$. A primary function of this experiment is to identify the plasma frequency and hence determine the electron density. The plasma frequency can be determined both by active stimulation of the resonance by a radio transmitter, and by observation of natural waves. In the active mode the time resolution is of the order of one minute, whilst in the natural waves mode it is a few seconds.

Higher time resolution measurements of the electron density may be achieved making use of the low frequency measurements of the spacecraft potential. Whilst the difference in the potential between two spherical probes is used to measure the electric field, the potential of a probe relative to the spacecraft structure measures the potential of the spacecraft relative to the plasma. The average of either two or four probe measurements may be used to reduce the influence of electric fields. The spacecraft potential is established as a result of the balance between emission of photo-electrons, and the return of electrons attracted by the positive structure. It depends in a complex way on the solar illumination, and the plasma composition, temperature and density. Over a short time scale it may be used to infer variations in the electron density between the measurements by WHISPER (Pedersen et al., 2001), or the electron analyser PEACE (Johnstone et al., 1997). The theoretical justification for the use of spacecraft potential data has been provided in many studies and interested reader should refer to the comprehensive explanation given in Pedersen et al. (2008). The main advantage is the increase in time resolution, from $\sim 4 \mathrm{~s}$ for PEACE, 1-2 s for WHISPER, but $0.2 \mathrm{~s}$ from the spacecraft potential. Thus it is possible to investigate structures in the electron density that are associated with the ducting of chorus emissions. In this section examples of the occurrence of chorus emissions observed on 23 August 2003 between 22:40 and 23:00 UT are described. During this period, the magnetic field magnitude was $~ 307 \mathrm{nT}$ implying an electron gyrofrequency of the order $8.6 \mathrm{kHz}$.

\subsection{Event 1 22:47:45}

The first event analysed occurred around 22:47:45 on 23 August 2003. The WBD dynamic spectra for the period 22:47:30-22:48:00 UT are shown in Fig. 1. The single band of activity at $\sim 2000 \mathrm{~Hz}$ is probably due to hiss. Its amplitude appears to pulse throughout the period of observations, an artifact of the $4 \mathrm{~s}$ spin period of the spacecraft. This appears to some extent in the spacecraft potential measurements but should not affect our conclusions as the periods of interest occur on much smaller time scales. A burst of chorus activity is observed at around 22:47:45 UT. It can clearly be seen that there are three or four intense rising tones in the frequency range $1.5-2.5 \mathrm{kHz}$ in the dynamic spectrogram, lasting for less than $0.75 \mathrm{~s}$. Since the frequency of the chorus is less than half the electron gyrofrequency it is possible for the

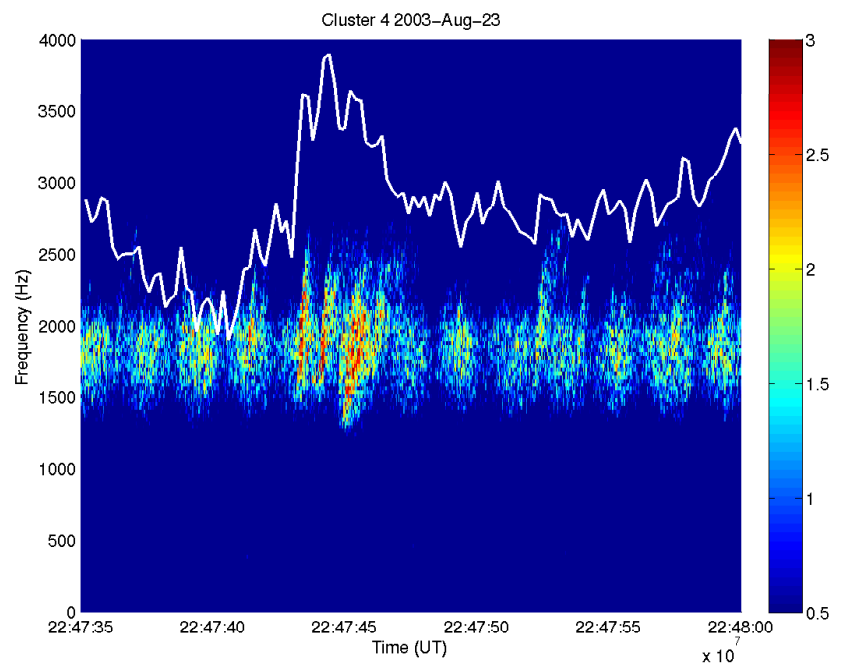

Fig. 1. Dynamic spectra of wave observations by the Cluster 4 WBD instrument for the period 22:47:30-22:28:00 UT. The white line represents the spacecraft potential and is used as a proxy measurement for the plasma electron density (suitably scaled).

waves to be trapped by both localised density enhancements and depletions.

The white line overplotted on Fig. 1 shows the (scaled) spacecraft potential measurements $\left(-V_{\mathrm{sc}}\right)$ from the EFW instrument. The more positive the values of $-V_{\mathrm{sc}}$ the more dense the plasma. The potential itself typically has a value of $-3.79 \mathrm{~V}$ and in the period shown varies in the range from -3.84 to $-3.74 \mathrm{~V}$. The least negative values of the potential, which correspond to the highest densities, are observed around 22:47:45, coincident with the observations of the chorus tones. This increase in the spacecraft potential contains four peaks that would indicate four regions of enhanced density. Thus, around this time it appears that Cluster 4 traverses regions of enhanced density in which the chorus emissions are observed. The first two rising tones show a simple, linear structure contained with in relatively narrow density enhancements. The third chorus emission appears to be single wave at frequencies $f<1.5 \mathrm{kHz}$ contained within a single density enhancement. However, at frequencies $f \sim 1.7 \mathrm{kHz}$ the observed waves appear to merge with the fourth (and smaller) density enhancement, possibly indicating that waves may pass from one duct to the next because they are separated by a region in which the spacecraft potential dips only slightly and hence may be unable to contain the waves in the two separate density enhancement regions. However, at frequencies $f>2000 \mathrm{kHz}$ the two chorus emissions appear to be confined to separate density enhancement regions. A closer inspection of this plot shows that there are other chorus emissions that are coincident with a peak in the spacecraft potential. The burst that occurred around 22:48:05 appears to have a similar, complex form to the third burst mentioned above. 


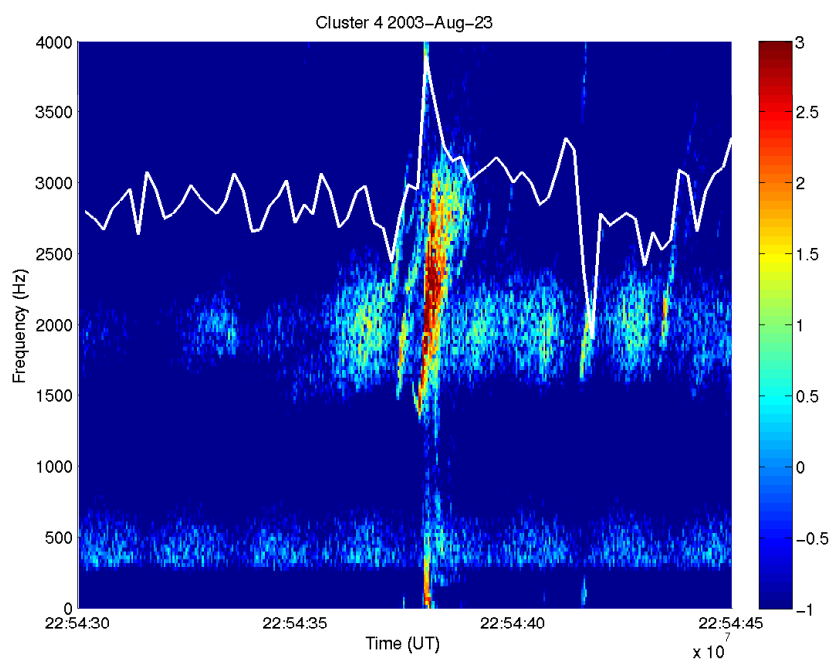

Fig. 2. Dynamic wave spectra and spacecraft potential plotted in the same format as Fig. 1 for the period 22:54:30-22:54:45 UT.

\subsection{Event 2 22:54:37}

Figure 2 shows a number of rising chorus emissions observed by WBD around 22:54:37 UT. The most intense risers begin at frequencies typically $1.8 \mathrm{kHz}$ and below and with upper limits of $>3 \mathrm{kHz}$. These emissions occur at times for which the spacecraft potential shows a peak, indicating a density enhancement. The largest amplitude chorus emission occurs at 22:54:38, corresponding to the time at which the largest fluctuation in the spacecraft potential occurs. The leading edge of this peak exhibits a large change in the potential, and thus a sharp density gradient. This increase in the potential is not continuous which would indicate that there may be two separate regions of increased density separated by a narrow trough with a slightly lower density. Further evidence for this complex structure is borne out by the occurrence of several chorus bands with different characteristics. At the onset of this increase in the spacecraft potential (22:54:37.18) a single chorus band appears, beginning at a frequency of $2.3 \mathrm{kHz}$ and ending around $3.2 \mathrm{kHz}$ at a time corresponding to the local peak in the potential (22:54:37.61). The gradient of this tone is approximately $2.35 \mathrm{kHz} \mathrm{s}^{-1}$. During this period of enhanced potential a second riser is observed at lower frequencies, beginning at $1.45 \mathrm{kHz}$ and ending around $2.1 \mathrm{kHz}$ at a time corresponding to the local potential maxima (gradient $4.7 \mathrm{kHz} \mathrm{s}^{-1}$ ). Following this initial enhancement, the potential drops slightly. During this period (22:54:37.61-22:53:37.76) a rising tone is observed in the frequency range $2.24-2.45 \mathrm{kHz}$. In contrast to the two previously observed risers, this tone has a considerably lower gradient, only $0.7 \mathrm{kHz} \mathrm{s}^{-1}$. After this there is another sharp increase in the potential. Once again, the peak in the potential corresponds to the onset of the most intense emissions seen in this event which span the frequency range $1.3-3.1 \mathrm{kHz}$. This burst probably contains many risers that cannot be resolved into individual components.

\section{Discussion and conclusions}

In the present paper we have reported cases when observation of increased chorus wave power in the low frequency band coincide with density crests as indicated by measurements of the spacecraft potential. As mentioned above it is well known that density enhancements, elongated along the magnetic field, can trap whistler waves at frequencies below the half electron gyrofrequency whilst density troughs can trap waves both above and below the half electron gyrofrequency.

The physics and theory of the generation of field aligned density irregularities attracted significant attention during the early stages of magnetosphere-ionospheric research. A number of early models were based on MHD instabilities (e.g. convective, "quasi-interchange" etc.) (Gold, 1959; Newcomb, 1961). A number of kinetic models were developed later. For example Richmond (1973) showed how pressure gradients can lead to the generation of field-aligned density irregularities. The theoretical model proposed by Bell and Ngo (1988) demonstrated how the amplification of chorus whistler waves can occur due to duct formation. In their model the conversion of whistler mode waves into lowerhybrid waves leads to increased pitch angle scattering and precipitation of radiation belt particles, resulting in a density enhancement in the ionosphere that forms field aligned density irregularities due to the upward diffusion of cold plasma. An overview of various theoretical mechanisms for the formation of field aligned density irregularities can be found in Carpenter et al. (2002). Implicit experimental evidence for the existence of ducts in the region of chorus waves have been reported on a number of occasions, for example Carpenter et al. (2002) analysed data from Radio Plasma Imager experiment on the IMAGE satellite. At lower altitudes it was shown how active ionospheric experiments can lead to the formation of ducts (Milikh et al., 2010).

In the present paper a few cases of simultaneous observations of chorus waves within the radiation belt and density enhancements were shown, indicating ducted propagation. A comprehensive statistical study of the ducted versus non-ducted propagation of chorus is currently being carried out and will be reported in future. It must be pointed out that even at the present stage it is obvious that not all chorus waves propagate in the ducted regime. It can be seen from Fig. 2 that lower amplitude chorus waves are observed outside density enhancements. The result that a significant number of high amplitude chorus waves propagate in the ducted mode imply that significant modifications should be implemented in presently used numerical models. For models based on the Fokker-Plank equation ducted propagation can both simplify and complicate the problem. On the one hand 
even for waves with oblique wave vectors, integration can be limited to a particular L-shell since despite the obliqueness of the wave propagation, the waves are still trapped on a particular field line by the density duct. On the other hand, ducted propagation complicates the relation between the effective wave parallel velocity and the simultaneous wave vector, because the velocity will also depend upon the geometry of the density irregularity. In the case of PIC simulations modifications are also required to either externally impose duct structures or by addressing their formation according to the previously proposed models. Summarising evidence of ducted propagation must have important implications for theoretical and numerical models of acceleration processes associated with the radiation belts.

Acknowledgements. The Sheffield authors wish to acknowledge financial support from STFC. YK is supported by the Swedish Research Council, grant 2007-4377. MAB wishes to thank G. Milikh for useful discussions.

Guest Editor M. Gedalin thanks one anonymous referee for her/his help in evaluating this paper.

\section{References}

Agapitov, O., Krasnoselskikh, V., Zaliznyak, Yu., Angelopoulos, V., Le Contel, O., and Rolland, G.: Chorus source region localization in the Earth's outer magnetosphere using THEMIS measurements, Ann. Geophys., 28, 1377-1386, doi:10.5194/angeo28-1377-2010, 2010.

Bell, T. F. and Ngo, H. D.: Electrostatic waves stimulated by coherent VLF signals propagating in and near the inner radiation belt, J. Geophys. Res., 93, 2599-2618, doi:10.1029/JA093iA04p02599, 1988.

Burton, R. K. and Holzer, R. E.: The Origin and Propagation of Chorus in the Outer Magnetosphere, J. Geophys. Res., 79, 10141023, doi:10.1029/JA079i007p01014, 1974.

Carpenter, D. L., Spasojević, M. A., Bell, T. F., Inan, U. S., Reinisch, B. W., Galkin, I. A., Benson, R. F., Green, J. L., Fung, S. F., and Boardsen, S. A.: Small-scale field-aligned plasmaspheric density structures inferred from the Radio Plasma Imager on IMAGE, J. Geophys. Res. (Space Physics), 107, 1258, doi:10.1029/2001JA009199, 2002.

Décréau, P. M. E., Fergeau, D., Kranosels'kikh, V., Lévêque, M., Martin, P., Randriamboarison, Sené, F. X., Trotignon, J., Canu, P., Mögensen, P., and Investigators, W.: WHISPER, A Resonance Sounder and Wave Analyser: Performances and Perspectives for the Cluster Mission, Space Sci. Rev., 79, 157-193, 1997.

Gold, T.: Motions in the Magnetosphere of the Earth, J. Geophys. Res., 64, 1219-1224, doi:10.1029/JZ064i009p01219, 1959.

Gurnett, D. A., Huff, R. L., and Kirchner, D. L.: The Wide-Band Plasma Wave Investigation, Sp. Sci. Rev., 79, 195-208, 1997.

Gustafsson, G., Bostrom, R., Holback, B., Holmgren, G., Lundgren, A., Stasiewicz, K., Ahlen, L., Mozer, F. S., Pankow, D., Harvey, P., Berg, P., Ulrich, R., Pedersen, A., Schmidt, R., Butler, A., Fransen, A. W. C., Klinge, D., Falthammar, C.-G., Lindqvist, P.-A., Christenson, S., Holtet, J., Lybekk, B., Sten, T. A., Tanskanen, P., Lappalainen, K., and Wygant, J.: The Electric Field and Wave Experiment for the Cluster mission, Space Sci. Rev., 79, 137-156, 1997.

Gustafsson, G., André, M., Carozzi, T., Eriksson, A. I., Fälthammar, C.-G., Grard, R., Holmgren, G., Holtet, J. A., Ivchenko, N., Karlsson, T., Khotyaintsev, Y., Klimov, S., Laakso, H., Lindqvist, P.-A., Lybekk, B., Marklund, G., Mozer, F., Mursula, K., Pedersen, A., Popielawska, B., Savin, S., Stasiewicz, K., Tanskanen, P., Vaivads, A., and Wahlund, J.-E.: First results of electric field and density observations by Cluster EFW based on initial months of operation, Ann. Geophys., 19, 1219-1240, doi:10.5194/angeo-19-1219-2001, 2001.

Helliwell, R. A., Crary, J. H., Pope, J. H., and Smith, R. L.: The "Nose" Whistler-A New High-Latitude Phenomenon, J. Geophys. Res., 61, 139-142, doi:10.1029/JZ061i001p00139, 1956.

Horne, R. B., Thorne, R. M., Glauert, S. A., Meredith, N. P., Pokhotelov, D., and Santolìk, O.: Electron acceleration in the Van Allen radiation belts by fast magnetosonic waves, Geophys. Res. Lett., 34, L17107, doi:10.1029/2007GL030267, 2007.

Johnson, M. H. and Kierein, J.: Combined release and radiation effects satellite (CRRES) - Spacecraft and mission, J. Spacecraft and Rockets, 29, 556-563, doi:10.2514/3.55641, 1992.

Johnstone, A. D., Alsop, C., Burge, S., Carter, P. J., Coates, A. J., Coker, A. J., Fazakerley, A. N., Grande, M., Gowen, R. A., Gurgiolo, C., Hancock, B., Narheim, B., Preece, A., Sheather, P., Winningham, J. D., and Woodliffe, R. D.: Peace: A Plasma Electron and Current Experiment, Space Sci. Rev., 79, 351-398, 1997.

Milikh, G. M., Demekhov, A. G., Papadopoulos, K., Vartanyan, A., Huba, J. D., and Joyce, G.: Model for artificial ionospheric duct formation due to HF heating, Geophys. Res. Lett., 370, L07803, doi:10.1029/2010GL042684, 2010.

Newcomb, W. A.: Convective Instability Induced by Gravity in a Plasma with a Frozen-In Magnetic Field, Phys. Fluids, 4, 391396, doi:10.1063/1.1706342, 1961.

Ni, B., Thorne, R. M., Meredith, N. P., Horne, R. B., and Shprits, Y. Y.: Resonant scattering of plasma sheet electrons leading to diffuse auroral precipitation: 2. Evaluation for whistler mode chorus waves, J. Geophys. Res. (Space Physics), 116, A04219, doi:10.1029/2010JA016233, 2011.

Pedersen, A., Décréau, P., Escoubet, C.-P., Gustafsson, G., Laakso, H., Lindqvist, P.-A., Lybekk, B., Masson, A., Mozer, F., and Vaivads, A.: Four-point high time resolution information on electron densities by the electric field experiments (EFW) on Cluster, Ann. Geophys., 19, 1483-1489, doi:10.5194/angeo-19-14832001, 2001.

Pedersen, A., Lybekk, B., André, M., Eriksson, A., Masson, A., Mozer, F. S., Lindqvist, P.-A., Décréau, P. M. E., Dandouras, I., Sauvaud, J.-A., Fazakerley, A., Taylor, M., Paschmann, G., Svenes, K. R., Torkar, K., and Whipple, E.: Electron density estimations derived from spacecraft potential measurements on Cluster in tenuous plasma regions, J. Geophys. Res. (Space Physics), 113, A07S33, doi:10.1029/2007JA012636, 2008.

Richmond, A. D.: Self-induced motions of thermal plasma in the magnetosphere and the stability of the plasmapause, Radio Sci., 8, 1019-1027, doi:10.1029/RS008i011p01019, 1973.

Sagdeev, R. Z. and Galeev, A. A.: Nonlinear Plasma Theory, Benjamin, White Plains, N.Y., 1969.

Santolik, O. and Gurnett, D. A.: Transverse dimensions of chorus in the source region, Geophys. Res. Lett., 30, 1031, 
doi:10.1029/2002GL016178, 2003.

Santolík, O., Gurnett, D. A., Pickett, J. S., Parrot, M., and Cornilleau-Wehrlin, N.: Central position of the source region of storm-time chorus, Planet. Sp. Sci., 53, 299-305, doi:10.1016/j.pss.2004.09.056, 2005.

Santolík, O., Gurnett, D. A., Pickett, J. S., Chum, J., and CornilleauWehrlin, N.: Oblique propagation of whistler mode waves in the chorus source region, J. Geophys. Res. (Space Physics), 114, A00F03, doi:10.1029/2009JA014586, 2009.

Shklyar, D. R.: On the nature of particle energization via resonant wave-particle interaction in the inhomogeneous magnetospheric plasma, Ann. Geophys., 29, 1179-1188, doi:10.5194/angeo-291179-2011, 2011a.

Shklyar, D. R.: Wave-particle interactions in marginally unstable plasma as a means of energy transfer between energetic particle populations, Phys. Lett. A, 375, 1583-1587, doi:10.1016/j.physleta.2011.02.067, 2011b.

Shklyar, D. R. and Kliem, B.: Relativistic electron scattering by electrostatic upper hybrid waves in the radiation belts., J. Geophys. Res., 111, A06204, doi:10.1029/2005JA011345, 2006.
Shklyar, D. and Matsumoto, H.: Oblique Whistler-Mode Waves in the Inhomogeneous Magnetospheric Plasma: Resonant Interactions with Energetic Charged Particles, Surv. Geophys., 30, 55104, doi:10.1007/s10712-009-9061-7, 2009.

Shprits, Y. Y., Thorne, R. M., Horne, R. B., and Summers, D.: Bounce-averaged diffusion coefficients for field-aligned chorus waves, J. Geophys. Res. (Space Physics), 111, A10225, doi:10.1029/2006JA011725, 2006.

Smith, R. L., Helliwell, R. A., and Yabroff, I. W.: A Theory of Trapping of Whistlers in Field-Aligned Columns of Enhanced Ionization, J. Geophys. Res., 65, 815, doi:10.1029/JZ065i003p00815, 1960.

Storey, L. R. O.: An Investigation of Whistling Atmospherics, Royal Society of London Philosophical Transactions Series A, 246, 113-141, doi:10.1098/rsta.1953.0011, 1953.

Woolliscroft, L. J. C., Alleyne, H. S. C., Dunford, C. M., Sumner, A., Thompson, J. A., Walker, S. N., Yearby, K. H., Buckley, A., Chapman, S., and Gough, M. P.: The Digital Wave-Processing Experiment on Cluster, Space Sci. Rev., 79, 209-231, 1997. 